\title{
Estrategias de crecimiento empresarial aplicadas por hipermercados*
}

\author{
Leal Morantes, Miraidy** \\ Cefalá Chirinos, Yilian***
}

\begin{abstract}
** Licenciada en Contaduría Pública, Magíster en Gerencia de Empresas. Investigadora del Proyecto Sistemas de Información en Super e Hipermercados. Docente de la Escuela de Administración y Contaduría Pública de la Facultad de Ciencias Económicas y Sociales de la Universidad del Zulia, Venezuela. E-mail: $\underline{\text { mylealm@yahoo.es }}$

*** Licenciada en Contaduría Pública, Maestrante del Programa de Gerencia de Empresas. Auxiliar de Investigación del Proyecto Sistemas de Información en Super e Hipermercados, Venezuela. E-mail: yiliancefala@yahoo.com
\end{abstract}

\section{Resumen}

El presente estudio tiene como objetivo caracterizar las estrategias de crecimiento empresarial, representadas por alianzas y adquisiciones, que han sido aplicadas en el sector de distribución de bienes de consumo masivo, específicamente en los hipermercados ubicados en Maracaibo, Estado Zulia, como manera de responder al entorno competitivo y cambiante en el cual se desenvuelven. La investigación se desarrolló a través de un estudio de carácter descriptivo; para la recolección de datos se aplicó un cuestionario estructurado, bajo la modalidad de entrevista, a gerentes generales y financieros. Los resultados evidencian que las estrategias de crecimiento empresarial utilizadas son la apertura de nuevos establecimientos comerciales, adquisición de empresas y participación accionaria en grandes cadenas, las cuales propician la concentración del mercado de distribución comercial, afectando a algunas empresas venezolanas del sector cuya oferta no puede competir; asimismo, los proveedores se enfrentan a cadenas con gran poder de negociación.

Palabras clave: Crecimiento empresarial, estrategias, hipermercados.

\section{Business Growth Strategies Applied to Hypermarkets}

\section{Abstract}

The objective of this study is to characterize business growth strategies, represented by 
alliances and acquisitions, and which have been applied to the mass consumption consumer goods market, specifically hypermarkets located in Maracaibo, Zulia State as a manner of responding to the dynamic competitive environment in which they operate. This research includes a descriptive study in which the collection of data was ucarried out using a structured questionaire in an interview with general and financial managers. The results indicate that business growth strategies utilized include the opening of new commercial outlets, the acquisition of similar businesses and stock participation in large chain operations, all of which stimulate commercial market concentration, resulting in the closing of other Venezuelan firms which cannot compete. Suppliers must face operative chains with great purchase power.

Key words: Business growth, strategies, hypermarkets.

Recibido: 03-02-04. Aceptado: 03-09-17

\section{I ntroducción}

Nuestras organizaciones se desenvuelven actualmente en un medio cambiante; caracterizado por avances tecnológicos, transformaciones políticas, sociales y económicas, que producen modificaciones en sus estructuras organizativas y exigen la reformulación de sus estrategias gerenciales, como manera de responder a las exigencias de un entorno cada vez más competitivo, incierto y globalizado. El sector de la distribución de bienes de consumo masivo ha sido uno de los sectores afectados por esos procesos de cambio y es el que ha respondido más rápidamente ante los mismos.

Tal como plantea Villalonga (1996: 1), en los últimos años la internacionalización del comercio ha experimentado una aceleración sin precedentes; esto ha sido propiciado, entre otros factores, por "la relativa saturación de los mercados nacionales, las barreras de entrada establecidas por los gobiernos nacionales/autonómicos y las limitaciones en volumen del mercado... (lo cual originó) que las principales empresas de distribución de Europa, Estados Unidos y Japón decidieran extenderse a países con alto potencial de crecimiento".

Otro factor que incidió favorablemente para la mundialización de la distribución fue la exportabilidad de algunos tipos de establecimientos comerciales: hipermercados y tiendas de descuento (Villalonga, 1996: 3). Es así como durante los últimos años las cadenas de hipermercados Carrefour, Makro, Wal Mart y Casino se han instalado en diferentes ciudades asiáticas y latinoamericanas; la cadena europea Carrefour decidió invertir en 
Colombia y Makro, entre los años ochenta y noventa, incursionó en Tailandia, Taiwán, Indonesia, Malasia, China y Filipinas (Makro Asia Management, 2002).

América Latina se ha convertido en un objetivo de suma importancia dentro las estrategias de expansión y consolidación de varias cadenas de establecimientos comerciales del tipo hipermercados, ya que hasta ahora esta región se constituía como un mercado cautivo con gran potencial para la incursión de las mismas.

En Venezuela, la cadena holandesa Makro, la cadena francesa Casino y la colombiana Éxito son algunos de los actores que han llegado para competir en el sector, con lo cual se prevé una mayor participación en los próximos años, originando cambios radicales en el comercio dedicado al consumo masivo y nuevos retos para el mundo empresarial venezolano.

Este nuevo escenario plantea la necesidad de buscar una reorientación en el manejo de las empresas existentes y un cambio en sus estrategias: incorporación de nuevos productos y servicios, fusiones, alianzas, búsqueda de socios estratégicos, entre otras. De la misma forma, se debe aprovechar el desarrollo tecnológico en informática e incrementar las inversiones en infraestructura y plataforma tecnológica para enfrentar la incursión en el mercado local de hipermercados internacionales.

Bajo el contexto anterior, en la presente investigación se identifican las acciones gerenciales adoptadas por los hipermercados ubicados en Maracaibo, Estado Zulia, con el fin de determinar cuáles predominan y cómo inciden en las empresas venezolanas, en función de la búsqueda de una mayor competitividad.

Para la recolección empírica de datos se aplicó, a gerentes generales y financieros, un cuestionario estructurado. Por otra parte, se realizaron entrevistas no estructuradas a personal administrativo clave de los hipermercados, a fin de obtener información adicional.

\section{Hipermercados en un entorno competitivo}

El nuevo escenario que plantea el mercado mundial y el incremento de la competencia de empresas extranjeras, especialmente en el sector de distribución, produce diversos impactos en las organizaciones, lo cual les exige la reformulación de sus estrategias gerenciales. 
Venezuela no es ajena a esta situación; en los últimos años ha experimentado la incursión de algunas cadenas de hipermercados internacionales, originando cambios en el comercio de bienes de consumo masivo y presiones competitivas en el sector privado.

Al respecto, es necesario destacar diversas investigaciones realizadas en nuestro país por la encuestadora Datos Information Resources (1998), relacionadas con la comercialización. Específicamente, en el año 1998 presentó el estudio "Story Audit Nacional" en el cual explica el cambio experimentado en la estructura de la comercialización nacional, pronosticando dicho estudio mayor participación de los hipermercados y supermercados para los próximos años.

Frente a este nuevo escenario, las empresas requieren formular estrategias de financiamiento y tomar decisiones de inversión acertadas que les ayuden a consolidarse y enfrentar el desafío de un mayor crecimiento dentro de un contexto de apertura económica y la búsqueda de posiciones competitivas en el mercado.

\subsection{Origen y características de los hipermercados}

La fórmula del hipermercado nace en Francia en el año 1963, cuando Marcel Fournier, Denis y Jacques Defforey, fundadores de Carrefour, inauguran un nuevo tipo de establecimiento de $2.500 \mathrm{~m} 2$ en Ste. Geneviéve des Bois, caracterizado por ofrecer una variedad de productos (alimentos y no alimentos), precios "discount" o con descuento y un mínimo de servicio (Aecoc, 1999).

Estos establecimientos tuvieron gran éxito en Europa y, posteriormente, empresas de distribución francesas y británicas intentan desarrollarlos en Estados Unidos sin obtener el éxito esperado, debido a que "el formato hipermercado era demasiado grande y el número de hipermercados demasiado pequeño para que la fórmula se generalizara y tuviera éxito"; sin embargo, años después buscaron la expansión a otros países (Villalonga, 1996: 3).

En Venezuela, los hipermercados surgen en la década de 1990; específicamente la cadena internacional Makro inicia sus operaciones en 1992프, pero es el año 1994 cuando comienza sus actividades comerciales en Maracaibo. Asimismo, el concepto de hipermercado de Super Maxy's fue lanzado a finales de 1995 en las ciudades de Caracas y Maracaibo (www.cativen.com; 13/10/2000).

De acuerdo con lo señalado por Omar Hidalgo (2001), Director de Finanzas de la cadena 
Makro, Venezuela es un polo atractivo para promover inversiones de este tipo, con la concurrencia de capital nacional y foráneo, ya que en nuestro país "hay espacio suficiente para la incursión de otras cadenas de establecimientos similares".

Actualmente, Makro cuenta con 17 tiendas ubicadas en diferentes ciudades del país y en el caso de Super Maxy's, su concepto original de hipermercados caracterizado por amplios espacios con la mayor variedad de productos y servicios, fue reorientado. En efecto, el establecimiento ubicado en la ciudad de Maracaibo fue reinaugurado en abril de 2002 bajo el nombre de Cada Supermercados (Diario Panorama, 05/06/2002: 3-9).

Construcentro, perteneciente al Grupo Corimon, fue otra de las cadenas de hipermercados presentes en el país. En los últimos años, este grupo experimentó pérdidas y un aumento considerable en los niveles de endeudamiento, por lo cual, como parte de su reestructuración y para disminuir sus pasivos, vendió los activos de la red a la Cadena Cativen-Casino por 39,5 millones de dólares, los cuales funcionarían bajo un nuevo formato de hipermercados (Diario Panorama, 03/07/2000: 2-5). Es así como la cadena Éxito comienza sus operaciones en Venezuela con la apertura de su primer local en Caracas, luego de la adquisición de los locales donde funcionó Construcentro, como parte de los planes de la Cadena de Tiendas Venezolanas (CATIVEN) ?

Luego de esta operación se comienza a observar la presencia de Éxito en 22 ciudades del país con el formato de supermercados (los supermercados CADA's) y en 4 ciudades del país operan también como hipermercados, siendo Maracaibo la única ciudad hasta el momento en contar con dos establecimientos de este último tipo (Diario Panorama, 17/11/2002: 3-2).

Actualmente, el número de hipermercados en el país se ha incrementado, aunque en forma incipiente; sin embargo, se observa el uso indiscriminado e inadecuado del término en algunos establecimientos que se autodenominan "hipermercados" sin reunir las características esenciales que definen este tipo de modelo de distribución comercial. Incluso, algunos no poseen las características básicas requeridas como son: amplios espacios de ventas y puestos de estacionamiento, así como variedad y surtido de productos y servicios. El empleo inadecuado del término puede deberse, como plantean Diez y Fernández (1993: 138), al desconocimiento de estas características por parte de los propietarios o simplemente lo utilizan porque resulta un nombre más atractivo para los consumidores.

Tomando en consideración los planteamientos de autores como Kotler (1993), Hasty y 
Reardon (1998), Meyer et al. (1992), Stern et al. (1999), las principales características que definen a nivel internacional a los hipermercados son las siguientes:

- "Superficies de grandes dimensiones. Al respecto, no existe un consenso general sobre las medidas de estos establecimientos; sin embargo, los autores coinciden en señalar superficies mayores a $10.000 \mathrm{~m} 2$.

- "Ofrecen "todo bajo un mismo techo". Tienen una amplia variedad de productos, incluyendo alimentos y no alimentos. Entre ellos se encuentran: ropa, calzados, muebles, electrodomésticos, medicamentos, línea blanca, videos, artículos de higiene y para el hogar, alimentos (víveres, frutas, carnes, verduras, lácteos), accesorios de automóviles, juguetería, artículos deportivos, ferretería, entre otros.

- "Ofrecen servicios adicionales como: cafeterías, restaurantes, pizzerías, panaderías, guarderías, ópticas, correo, taquillas de pago de servicios, agencias de viajes, cajeros automáticos, gasolineras.

- "Poseen amplios estacionamientos.

- "Poseen amplios pasillos con estanterías altas, tipo dep ósito, para la exhibición en conjunto del inventario.

- "Realizan compras en gran volumen y tienen alta rotación de inventarios.

- "Ofrecen productos de marcas propias o marcas del distribuidor.

- "Ofrecen precios bajos.

- "Presentan bajo margen de rentabilidad.

- "Tienen modernos y sofisticados sistemas computarizados.

- "Tienen horarios extendidos.

- "Proporcionan mínima atención al cliente, operan bajo el esquema de autoservicio.

Generalmente, los hipermercados son propiedad de grandes grupos financieros o de poderosas cadenas de distribución multinacionales, debido a la magnitud económica que implica la instalación del establecimiento; sus ventajas radican en altos volúmenes de ventas, capacidad para comprar en grandes cantidades y la posibilidad de tener cierto poder de negociación con sus proveedores que les permite obtener descuentos y economías de escala.

Según Joseph Saade (2001), vicepresidente ejecutivo de Datos Information Resources, en Venezuela se consideran hipermercados grandes superficies que ofrezcan una amplia gama de productos como alimentos, línea blanca, línea marrón, vestido y calzado.

Para Carlos Hugo Escobar (2001), gerente general de CATIVEN, los hipermercados se caracterizan por: área de ventas superior a los $5.000 \mathrm{~m} 2$; servicios de empacado, 
transporte y postventa; disponibilidad de una serie de productos que no suelen encontrarse en supermercados tradicionales; disponibilidad de productos variados, como: líneas de confección y lencería, línea digital (audio, video, sonido y computación) y comidas preparadas para llevar.

Actualmente los hipermercados y demás empresas del sector minorista están centrados en ofrecer, además de variedad de productos, una serie de servicios adicionales que añadan valor a su actividad principal, a fin de influir en la decisión de compra de los consumidores. Tal como plantean Casares y Rebollo (cit. por Cruz, 1999: 18) "la decisión de compra de los consumidores se fundamenta en la adquisición de una cesta de características que mejor satisfagan sus necesidades".

Al respecto, los hipermercados buscan mejorar su sistema de distribución en beneficio del consumidor para ofrecerles la posibilidad de comprar todos los productos en un mismo destino, con un buen nivel de servicio, conveniencia y precios bajos; una opción muy atractiva que ha permitido su penetración y participación progresiva en el mercado.

\subsection{Competencia en la distribución de bienes de consumo masivo}

La distribución de los bienes en el mercado se efectúa a través de los canales correspondientes, entendiéndose por canal "el sendero o camino específico elegido por el fabricante para hacer llegar sus productos al consumidor final" (Suárez, 1992: 54-55). Estos canales pueden estar compuestos por varios eslabones, dependiendo: del tipo de producto o bien que se comercialice, de los intermediarios, de las condiciones existentes en el mercado y en la economía en general. Si la propiedad del producto pasa directamente del fabricante al consumidor estaremos en presencia de un canal simple:

\section{Productor ${ }^{\circledR}$ Consumidor}

pero si la propiedad pasa a un comerciante para su posterior venta, el canal presenta un nuevo participante, alargándose el canal al tipo de intermediación:

Productor $\circledR^{\circledR}$ Intermediario ${ }^{\circledR}$ Consumidor

Este nuevo participante en el canal puede ser de dos tipos: mayorista o minorista, dependiendo si la venta de los bienes está destinada al uso comercial y/o reventa o si está destinada a satisfacer las necesidades de los consumidores finales, respectivamente. 
Los hipermercados, como intermediarios en la cadena de distribución, se mueven en dos escenarios: como clientes del sector industrial y agropecuario, demandando productos a los fabricantes, y como proveedores de oferta de productos y servicios a los consumidores finales. En este sentido, con base en los planteamientos de Cruz (1999), los hipermercados enfrentan un doble tipo de competencia: horizontal y vertical.

\subsubsection{Competencia horizontal con empresas similares}

Este tipo de competencia puede originarse entre empresas existentes que comercializan productos similares o con la entrada potencial de nuevas empresas en el sector. Cuando la competencia se produce entre diferentes tipos de establecimientos comerciales, tales como hipermercados, supermercados, tiendas por departamento, se conoce como competencia intertipo, y si ocurre dentro de un mismo tipo de establecimiento comercial, como por ejemplo, la competencia entre diferentes hipermercados, es conocida como competencia intratipo (Cruz, 1999).

En la competencia intertipo los diferentes establecimientos compiten entre sí para captar un mismo mercado, lo que origina que la cuota ganada por uno de ellos se obtenga en perjuicio de otro (Cruz, 1999). Esta situación se observa en Venezuela a partir del año 1997 cuando se produce una mayor participación de los hipermercados y supermercados en el comercio minorista en detrimento de los abastos y bodegas; en efecto, estudios realizados por Datanálisis (2001) confirman que más de 2.700 bodegas han cerrado en los últimos cuatro años.

\subsubsection{Competencia vertical}

La integración vertical es una estrategia que amplía el ámbito de acción competitiva de las organizaciones dentro de la misma industria; en el caso de los distribuidores, está representada por el poder de negociación que tengan frente a sus proveedores. En este sentido, el principal propósito de los hipermercados es la competitividad, para lo cual desarrollan economías de escala, optiman tiempos y costos operativos y, además, procuran la compra centralizada y al por mayor de la mercancía, así como el desarrollo de sociedades $\mathrm{y} / \mathrm{o}$ acuerdos con sus proveedores industriales, agrícolas y de servicios (Corporación Colombia Internacional, 1999).

Bajo este contexto, aquellos establecimientos o cadenas del sector que puedan disponer de un mayor grado de control de la demanda de los consumidores y, por lo tanto, de garantizar un mercado para los productos de los fabricantes, tendrán posibilidad de 
mejorar su posición negociadora frente a sus proveedores, lo que se traduce en exigencias de mejores condiciones en los contratos de compra de los productos, tales como: precios más bajos, mejores condiciones de pago, descuentos, condiciones de entrega, entre otros (Cruz, 1999); o la obtención de una ventaja o posición competitiva en el mercado.

Cuando se logra la integración vertical, se modernizan las operaciones de la compañía de forma que mejoran la flexibilidad organizacional, reducen los ciclos de tiempo, apresuran la toma de decisiones y reducen los costos de coordinación (Thompson y Strickland, 2001: 169).

En el caso venezolano, el poder de negociación que tienen las grandes cadenas está basado en las altas cuotas de participación en el mercado, lo cual permite obtener condiciones muy ventajosas en los contratos con sus proveedores. Entre los beneficios que obtienen los hipermercados se encuentran:

a) Obtención de plazos extensos para la cancelación de compras a crédito, como consecuencia de un proceso de integración vertical. Mediante esta estrategia las empresas buscan aumentar el control sobre los proveedores o adquirir el dominio sobre ellos (David, 1997: 54).

En los hipermercados objeto de estudio, el período promedio de pago es de 45 días, como mínimo, y en algunos casos 90 días, lo que produce la extensión de los plazos más allá de las necesidades reales de financiamiento del proceso de comercialización, constituyendo éste un ejemplo de algunas de las condiciones que imponen los hipermercados a las empresas para pertenecer a su cartera de proveedores. Al respecto, Rosales (1997: 4) establece que en este tipo de redes "el ámbito de la cooperación está determinado... por los intereses estratégicos de las empresas líderes".

b) Negociación de precios fijos para productos: Los hipermercados mantienen con sus proveedores acuerdos estables de aprovisionamiento y precios fijos (en algunos casos se establecen los precios para todo el año); en el caso de productos agrícolas, los precios se establecen con base en toda la cosecha.

c) Empleo de centrales de compra, para coordinar las solicitudes de las diferentes tiendas, lo cual permite obtener mejores condiciones de compra que aquellas que corresponderían a volúmenes individuales.

Adicionalmente, los hipermercados reciben otros beneficios de sus proveedores, como descuentos regulares por altos volúmenes de compra o inauguración de nuevos establecimientos comerciales, así como bonificaciones y contribuciones para ofertas 
vinculadas a ventas aniversario (Leal, 2003: 319).

\subsection{Estrategias de crecimiento empresarial para la distribución competitiva de bienes de consumo masivo}

Una de las principales funciones de la gerencia financiera consiste en maximizar el patrimonio de los accionistas de la organización, por lo cual debe estar familiarizada con el ambiente en el cual la empresa se desenvuelve y ser capaz de adaptarse al entorno cambiante, si quiere que ésta sobreviva.

Bajo este contexto, se ha producido un cambio en las decisiones de inversión; éstas no se limitan a determinar la cantidad total de activos necesarios para mantener la empresa, sino que en algunas ocasiones involucran procesos de reorganización o reestructuración, entendiéndose este proceso como "cualquier operación tendente a alterar la estructura de capital de una empresa, su capacidad productiva o su estructura de propiedad, siempre que no esté inmersa en el curso normal de los negocios de la misma" (Mascareñas, 1996: 311). En consecuencia, muchas empresas han adoptado estrategias de crecimiento de su tamaño, buscando reducir sus costos de distribución y aprovisionamiento, que les permitan responder a las exigencias del entorno.

Este crecimiento, realizado para expandir sus actividades, bien sea por la introducción de nuevos productos o líneas o por la penetración de nuevas áreas o mercados, puede ser llevado a cabo de dos formas (Cruz, 1999): a) crecimiento interno, basado en la apertura de nuevos establecimientos comerciales y b) crecimiento externo, mediante fusiones y adquisiciones de otras empresas, establecimiento de acuerdos de colaboración o alianzas estratégicas.

Esta segunda modalidad de crecimiento es la que ha tomado auge en el comercio minorista, principalmente en Europa, donde países como España han experimentado un fuerte proceso de fusiones y absorciones en la década de los noventa (Cruz, 1999).

En el caso venezolano, la cadena francesa Casino y la colombiana Éxito son los más recientes actores que han llegado al país para competir en el negocio de los hipermercados, cuyo crecimiento empresarial se ha llevado a cabo mediante la apertura de nuevos establecimientos comerciales a lo largo y ancho de la geografía nacional, adquisición de empresas y participación accionaria en grandes cadenas.

Makro, inversión conjunta de SHV Holanda (propietaria del 75\% del capital) y el Grupo 
Polar (con el $25 \%$ de las acciones), ha llevado a cabo su crecimiento empresarial mediante la apertura de nuevos establecimientos comerciales y actualmente cuenta con diecisiete (17) tiendas en toda Venezuela. Esta expansión ha sido financiada mediante el uso del crédito bancario y la reinversión de utilidades; asimismo, la emisión de papeles comerciales, con tres series exitosas, se utilizó en una sola oportunidad para financiar la apertura de dos de las tiendas ubicadas en el país.

La estrategia de abrir varios almacenes en zonas cercanas dentro de su propio país y contar con más de un punto de venta en cada país hacia el cual se extienden, evidencia que la tendencia en los planes corporativos de la mayoría de las empresas dedicadas al negocio de hipermercados es continuar ampliando su participación en el mercado.

La cadena francesa Casino, encargada de operar el Hipermercado Éxito en Venezuela, adquirió la mayoría de las acciones $(50,01 \%$ ) de CATIVEN (consorcio conformado también por la cadena colombiana Cadenalco y el Grupo Polar), por lo cual, además de los hipermercados, maneja la cadena de supermercados CADA.

Básicamente, el propósito que persigue la cadena con la apertura de nuevas tiendas y las adquisiciones es obtener economías de escala y eliminar competidores. Por ejemplo, el hipermercado Éxito reembolsa a sus clientes, bajo ciertas condiciones, la diferencia en el precio de algún producto que sea más económico en otro establecimiento competidor, ubicado en un perímetro no superior a 30 kilómetros.

Con relación a la reducción de costos, ésta se obtiene por disminución del costo de adquisición de los productos, al aumentar el volumen de compra. Los hipermercados utilizan centrales de compra para coordinar las solicitudes de las diferentes tiendas, lo cual permite obtener descuentos importantes por volúmenes comprados; asimismo, manejan centros de acopio para la recepción, procesamiento y distribución de los productos.

Otros hipermercados que han iniciado sus operaciones en Maracaibo son:

- "El hipermercado De Cándido, ubicado en la Avenida La Limpia, el cual cuenta con $10.000 \mathrm{~m} 2$, aproximadamente 20.000 ítems de productos y cuya construcción requirió una inversión superior a los 8.000 millones de bolívares (De Cándido, 2000: C-8).

- "El hipermercado Meta, que estuvo ubicado en el Centro Comercial "Centro Sur", el cual fue resultado de una alianza entre Promohiper, S.A. (compañía española propietaria del $95 \%$ del capital del hipermercado) y Casa París, S.A. (compañía 
venezolana con el $5 \%$ del capital) el cual, posteriormente, fue vendido a CATIVEN y en la actualidad, luego de mejoras sustanciales, opera bajo la denominación de Hipermercado Éxito. Al efecto, es importante destacar que a finales del año 2001 el grupo Casino celebró una alianza estratégica con el Grupo Martín Romero Asesores (GMR), corporación española dedicada a la construcción de centros comerciales. El acuerdo con la cadena de hipermercados establece que en todos los centros comerciales que construya el grupo se reservará un local para ella (Perozo, 2002: G-8).

Las alianzas surgen como una manera de unir esfuerzos para competir de una manera más efectiva, especialmente en tiempos de globalización, así como para aprovechar oportunidades que se presentan. Tal como lo plantea Peixoto (1998: 14) "... Acuerdos y asociaciones parecen marcar la transformación de la estrategia de las empresas frente a un ambiente económico de cambios e incertidumbre".

Las alianzas no sólo pueden compensar las desventajas competitivas, sino también dar por resultado que las compañías aliadas dirijan sus energías estratégicas más hacia los mutuos rivales y menos hacia sus aliados (Thompson y Strickland, 2001: 171).

Las alianzas son muy comunes para ingresar a mercados internacionales cuando se produce entre empresas nacionales y extranjeras, a la vez que permiten recabar recursos y acumular capacidades necesarias para hacer negocios en un mayor número de mercados foráneos.

En el caso de las alianzas entre empresas de distribución de productos de consumo masivo, generalmente se llevan a cabo para expandir la gama de productos, aumentar la base de clientes o para reducir costos de distribución o comercialización (Trigo y Drudis, 1999), señalando los autores que el área comercial es más propicia a la formalización de acuerdos horizontales.

La expansión empresarial de las empresas en Venezuela se ha realizado mediante la utilización del crédito bancario, principalmente para los hipermercados de capital nacional, sin embargo, las grandes cadenas internacionales han empleado para su expansión recursos generados por sus operaciones y los aportados por las empresas matrices (Leal, 2003: 317). Esta expansión se ha visto favorecida por los siguientes factores:

- "Gran potencial de crecimiento de la inversión nacional y extranjera en el sector debido a la escasa competencia existente en el modelo de hipermercados. 
- "Posibilidad de captar nuevos mercados debido a la desaparición de ciertas formas comerciales que resultan obsoletas, considerando los comportamientos y deseos actuales de los consumidores.

- "Incentivos fiscales que pueden obtenerse por nuevas inversiones, como por ejemplo, la reducción o exoneración de impuestos municipales.

Las estrategias de crecimiento empresarial de los hipermercados en nuestro país se han efectuado para aumentar la base de clientes, reducir costos de distribución, obtener economías de escala, asegurar distribución de productos y eliminar competencia; estas acciones están dirigidas a concentrar el mercado y son llevadas a cabo en detrimento de empresas regionales y nacionales. En efecto, Makro se estableció en el país en 1992 y en menos de 4 años pasó a ser la tercera empresa en volumen de ventas en Venezuela (Enac, 2001), con ventas anuales aproximadas de US \$ 700 millones (Empresas Polar, 2001) y CATIVEN es la cadena líder de supermercados en el país.

Las altas cuotas de participación en el mercado de las grandes cadenas, les permite disponer de un mayor control de la demanda de los consumidores y garantizar mercado para los bienes de fabricantes y productores; esta situación les confiere un gran poder de negociación frente a sus proveedores que les permite controlar las condiciones de la relación comercial, obteniendo condiciones muy ventajosas en los contratos de compra.

Por otra parte, algunas empresas venezolanas fabrican por encargo de los hipermercados productos a más bajo costo, que luego son comercializados como productos de marca propia a un precio menor que los artículos de otras marcas (aproximadamente un $25 \%$ de las ventas en los hipermercados estudiados son productos de marca propia).

Estos beneficios obtenidos por los hipermercados permiten reforzar su posición competitiva frente a otras empresas del sector, al ofrecer a los consumidores precios más bajos, competitivos, afectando a pequeños establecimientos y redes locales, cuya oferta no puede competir con la de grandes cadenas (Leal, 2003: 319). Al respecto, según un artículo publicado en la Revista Inversiones "... a finales de 1997 más del 37\% de los abastos y pequeños establecimientos habían desaparecido del mercado venezolano, a consecuencia del crecimiento de los hipermercados, por los cuales transitan diariamente más de 10 mil personas" (Barrios, 2000: 1); asimismo, estudios realizados por Datanálisis (2001) confirman que más de 2700 bodegas han cerrado en los últimos cuatro años, afectando considerablemente el nivel de empleo. 
Por otra parte, el éxito de los hipermercados en el mercado venezolano estaría propiciado por una serie de características que resultan atractivas a los consumidores: variedad y surtido de productos, precios bajos, como consecuencia de las grandes compras por volumen que realizan estos establecimientos, adecuada infraestructura y una serie de servicios adicionales que serían bien recibidos por aquellos consumidores que no disponen de mucho tiempo para realizar sus compras y prefieren efectuarlas en un solo lugar. Además, según diversos estudios llevados a cabo en el país por Datanálisis (2001) casi el $50 \%$ de los consumidores venezolanos realizan sus compras en supermercados y grandes cadenas buscando variedad, precios bajos y promociones.

Ante esta situación, las empresas venezolanas del sector, para poder competir o sobrevivir en el mercado, deberán establecer alianzas, asociaciones o acuerdos de colaboración para enfrentar mejor las exigencias, a la vez que les permitan ampliar el acceso al mercado, como consecuencia de mayores volúmenes de comercialización conjunta, y por lo tanto, obtener descuentos por volúmenes de compra (Leal, 2003: 321).

\section{Conclusiones}

La distribución de bienes de consumo masivo en Venezuela, ha experimentado una serie de transformaciones en los últimos años.

El crecimiento empresarial de los hipermercados, que comenzó tímidamente a principio de los años noventa con la incursión de la cadena internacional Makro (líder actualmente en el país) y posteriormente con otros grupos internacionales y locales, se ha llevado a cabo mediante la apertura de nuevos establecimientos y la adquisición de cadenas y empresas existentes.

Víveres De Cándido y Makro han llevado a cabo su estrategia de expansión empresarial mediante la apertura de nuevos establecimientos comerciales, efectuándose en este último caso con la participación de capital nacional y extranjero; la cadena Casino, encargada de operar el hipermercado Éxito en Venezuela, adquirió la mayoría de las acciones de CATIVEN y Éxito inició sus operaciones luego de la adquisición de la red de tiendas Construcentro.

Este proceso de crecimiento está dirigido a concentrar el mercado de distribución comercial y se realiza a costa de la quiebra de empresas regionales y nacionales o provocando procesos de reestructuración en las mismas, lo cual impone a las empresas locales adecuar su tamaño a los nuevos parámetros a fin de mantener su posición 
competitiva en el mercado, situación que se ve propiciada por las oportunidades económicas que ofrece Venezuela. Sin embargo, las condiciones favorables que pueda tener el país no es garantía suficiente para la promoción exitosa de dichas inversiones, afectado principalmente por el clima de incertidumbre político y económico reinante, por lo cual, corresponde al Gobierno Nacional crear condiciones que provean verdadera estabilidad y seguridad jurídica y, por lo tanto, genere mayor confianza en los inversionistas nacionales y extranjeros.

Por otra parte, la creciente penetración de las grandes tiendas de productos de consumo masivo produce efectos que se reflejan directamente en algunas empresas venezolanas del sector, ya que pequeños establecimientos y algunas cadenas locales, cuya oferta no puede competir con la de grandes empresas, han cerrado sus puertas, produciendo consecuencias negativas en el nivel de empleo. Asimismo, los proveedores se enfrentan a cadenas con gran poder de negociación que controlan las condiciones y términos de la relación comercial, obteniendo condiciones favorables en los contratos de compra, como consecuencia de un proceso de integración en red.

\section{Notas}

* Este trabajo constituye un producto del Proyecto de Investigación "Sistemas de Información en Super e Hipermercados", adscrito al Centro de Estudios de la Empresa de la Facultad de Ciencias Económicas y Sociales de la Universidad del Zulia y financiado por el Consejo de Desarrollo Científico y Humanístico.

1. Makro no responde totalmente al formato hipermercado; su oferta está dirigida al pequeño y mediano comerciante, mayorista y profesional independiente. Sin embargo, ha aprovechado las oportunidades del país para desarrollar este modelo de comercialización.

2. Cativen es un consorcio conformado por el grupo francés Casino que posee el $50,01 \%$ de participación, las cadenas colombianas Éxito-Cadenalco con el 27,99\% y el grupo venezolano Polar con el 22\% (Fernández, 2001).

\section{Referencias Bibliográficas}

1. Aecoc (1999), El Hipermercado se reinventa a sí mismo. http://www.aecoc.es/. Consulta: Julio $11 / 2000$.

2. Barrios Hernández, Belkis (2000), Mientras más grandes, mejor... Los negocios de consumo masivo replantean sus estrategias de crecimiento. Http:// www.InversionesVenezuela.com. Consulta: Septiembre 5/2000. 
3. Cativen (S/A), Supermaxy's. Http://www.cativen.com/supmaxys.htm. Consulta: Octubre 13/2000.

4. Corporación Colombia Internacional (1999), "Los hipermercados y sus tendencias en el Mundo y en Colombia. Boletín $\mathrm{CCl}$ ". Exótica. Año 3. Vol. 10. Marzo-Junio. Http: www.cci.org.co/publicaciones/Exotica/exotica10.html\#Mercados_Internacionales Consulta: Abril 30/2002.

5. Cruz Roche, Ignacio (1999), Los Canales de Distribución de Productos de Gran Consumo. Concentración y Competencia. España. Ediciones Pirámides, S.A

6. Datanálisis (2001), Venezolanos compran en supermercados o comercios informales. Http://www.datanálisis.com/ publicaciones/detalles.Asp? Cod=37 Consulta: Noviembre $26 / 2001$.

7. Datos Information Resources (1998), http://www.datosir.com. Consulta: Septiembre $05 / 2000$.

8. David, Fred R. (1997), Conceptos de Administración Estratégica. México. 5ta. Edición. Editorial Prentice Hall Hispanoamericana, S.A.

9. De Cándido, Andrés (2000), “Con la apertura del nuevo hipermercado DE CANDIDO aumentará $12 \%$ su participación en la región". Diario La Verdad, Sábado, 29 de Julio de 2000. C-8. Maracaibo, Venezuela.

10. Diario Panorama (03/07/2000), Corimon mira al pasado apostando a un éxito futuro. Maracaibo, Venezuela.

11. Diario Panorama (05/06/2002), Cada abrió sus puertas. Maracaibo, Venezuela.

12. Diario Panorama (17/11/2002). Éxito Abrió cinco tiendas en 15 meses. Hipermercados. En 2003 se remodelarán 30 Cada en 22 ciudades del país. Maracaibo, Venezuela.

13. Diez de Castro, Enrique C. y Fernández Fernández, Juan C (1993), Distribución Comercial. España. Mc Graw Hill.

14.

Empresas

Polar

(2001)， 
http://www.empresaspolar.com/nuevasinver/espanol/nuevas-dye-c.es. Consulta: Julio $26 / 2001$.

15. Enac (2001), http://www.eniac.com/notmakro.htm. Consulta: Octubre 23/2001.

16. Escobar, Carlos Hugo (2001), Establecimientos. Excelsior Gama: Gamaventas electrónicas/ Cativen: Preparando la invasión. Http://www.../articuloview.pl? listado=supermercado\& volumen=revista31\&codigo $=$ establecimientos\&encab $=00800$. Consulta: Julio 26/2001.

17. Fernández, Jainelly (2001), Cadena Éxito abrió en Maracaibo. Diario Panorama. Miércoles, 28 de Noviembre de 2001. 2-6. Maracaibo, Venezuela.

18. Hasty, Ron y Reardon, James (1998), Gerencia de Ventas al Detal. Colombia. Mc Graw Hill Interamericana, S.A.

19. Hidalgo, Omar (2001), “El Mercado Nacional Adopta Nuevos Cánones. Comerciar en Mayúsculas". Revista Venezuela Now - 4ta. Edición http://www.conapri.org/webespanol/r4-8e.html. Fecha de consulta: Octubre 23/2001.

20. Kotler, Philip (1993), Dirección de la Mercadotecnia. Análisis, Planeación, I mplementación y Control. México. 7ma. Edición. Prentice-Hall Hispanoamericana, S.A.

21. Leal Morantes, Miraidy (2003), “Estrategias de Financiamiento aplicadas por Hipermercados en un Entorno Competitivo". Revista de Ciencias Sociales. Vol. IX, No. 2. Venezuela. Universidad del Zulia, Instituto de Investigaciones. Pp 311-322.

22. Makro Asia Management (2002), http://www.makro.com/history.htm. Consulta: Abril 24/2002.

23. Mascareñas Pérez-Iñigo, Juan (1996), Manual de Fusiones y Adquisiciones de Empresas. España. 2da. Edición. Mc Graw Hill Interamericana de España, S.A.

24. Meyer, Warren G.; Harris, E. Edward; Kohns, Donald P. y Stone, James R. (1992), Marketing. Ventas al por Menor. Colombia. 8va. Edición. Mc Graw Hill Interamericana, S.A.

25. Peixoto de Albuquerque, Paulo (1998), "Las estrategias empresariales y el nuevo 
ambiente económico. La realidad virtual del cambio". En: Revista Venezolana de Gerencia. Año 3, No. 5. Maracaibo, Venezuela. Universidad del Zulia, Vice-Rectorado Académico. Pp 9-24.

26. Perozo, Fernando (2002), “Grupo Martín Romero Asesores prevé construir 7 centros comerciales". Diario EI Nacional. Domingo 7 de Abril 2002. G-8. Caracas, Venezuela.

27. Rosales, Ramón (1997), "La Asociatividad como estrategia de fortalecimiento de las PYMEs". Revista Capítulos. No. 51. Julio-Septiembre. Http://lanic.utexas. Edu/ sela/AA2K/ES/cap/N51/ rcap517.htm. Consulta: Febrero 11/2001.

28. Saade, Joseph (2001), Entrevista. Super, Hiper y conveniencia: formatos del nuevo milenio.http://www.global.../articuloview.pl? listado $=$ supermercado $\&$ volumen $=$ revista $31 \&$ codigo $=$ entrevista\&encab $=80400$. Consulta: Julio $26 / 2001$.

29. Stern, Louis W., El Ansary, Adel I., Coughlan Anne T., Cruz, Ignacio (1999), Canales de Comercialización. España. 5ta. edición. Prentice Hall Iberia, S.R.L.

30. Suárez Suárez, Andrés S. (1992), Diccionario de Economía y Administración. España. Mc Graw Hill-Interamericana de España, S.A.

31. Thompson, Arthur A. y Strickland A.J. (2001), Administración Estratégica. Conceptos y Casos. México. Mc- GrawHill.

32. Trigo, Joaquín y Drudis, Antoni (1999), Alianzas Estratégicas. Las claves y la práctica de la cooperación entre empresas. España. Ediciones Gestión 2000, S.A.

33. Villalonga, Magda (1996), La Globalización de los mercados en la Distribución. http://www. aecoc.es/. Consulta: Julio 11/2000. 